\title{
Complexity Results for Wireless Sensor Network Scheduling
}

\author{
Fethi Jarray \\ Laboratoire Cédric, Paris, France \\ Gabes University of Sciences, Gabes, Tunisia \\ Al-Imam Mohamed Ibn Saoud University, Riyadh, Saudi Arabia \\ E-mail: Fethi.jarray@cnam.fr \\ Received January 31, 2010; revised February 22, 2010; accepted February 23, 2010
}

\begin{abstract}
We study the problem of scheduling multiple sensors to visit and observe a group of sites at discrete time points over a planning horizon of given length. We show that scheduling under a given number of visits for each site and in each period is an NP-complete problem by providing equivalence with a problem in discrete tomography. We also give a polynomial time algorithm to schedule the sensors under a given number of visits in each period.
\end{abstract}

Keywords: Discrete Tomography, Sensor Scheduling, Combinatorial Optimization

\section{Introduction}

A wireless sensor is a small physical device having a battery with a limited capacity and having a transmission, a reception capability of limited range and capable to take various measurements of its neighborhood. These measurements include temperature, acoustic, solar radiation, etc. Since a single sensor is not able to monitor a hole field, a group of sensors should be deployed and frequently interact with each other to exchange information. This group of distributed sensors forms the wireless sensor networks (WSN). Generally, the basic goal of a WSN is to ensure the surveillance of a given region with a limited number of sensors and eventually transmit the sensed data to a processing unit.

The management and the organization of WSN have been investigated by many researchers. Depending on the application and the goal of researchers, several combinatorial optimization models and solution techniques have been proposed [1]. These models and problems include, among others, sensor localization and tracking [2-5], sensor scheduling [6-11] communication neutralization [12,13] and energy consumption [14,15]. There are a wide variety of methods and models because on one had several issues are considered in WSN. On the other hand, the most addressed problems are NP-complete, i.e., there is no polynomial time algorithm to solves these problems unless $\mathrm{P}=\mathrm{NP}$.

This paper is concerned with WSN scheduling to pe- riodically monitor and observe an environment. WSN scheduling is needed in various applications such as traffic monitoring at roads, military, medical, pollution detection, etc. In this paper, we show the complexity results of WSN scheduling by supposing that the number of required visits for each period are given. We will use a reduction from an independent problem of discrete tomography. For clarity, the next section is devoted to the area of discrete tomography.

The remainder of this paper is organized as follows. In Section 2, the problem of discrete tomography and binary matrices reconstruction will be presented. In Section 3, Sensor scheduling with a given numbers of visits per period and per site will be studied. In Section 4, sensor scheduling with a given numbers of visits per period is addressed.

\section{Discrete Tomography}

Discrete Tomography (DT) is an emergent area of computer science (refer to the books of Hermann and Kuba $[16,17]$ for further information on the theory, algorithms and applications). It deals with the reconstruction of binary matrices and images from horizontal and vertical projections. Let $\mathrm{A}$ be a binary matrix of size $\mathrm{m} \times \mathrm{n}$, we denote by $h_{i}=\sum_{j=1}^{n} A_{i j}$ the number of ones on row $i$ and by $v_{j}=\sum_{i=1}^{m} A_{i j}$ the number of ones on column $j$. 
The vectors $H=\left(h_{1}, \cdots, h_{m}\right)$ and $V=\left(v_{1}, \cdots, v_{n}\right)$ are called the horizontal and the vertical projection respectively. The problem of reconstructing a binary matrix from orthogonal projections, denoted $\operatorname{MB}(H, V)$, is defined as follows: given two vectors $H$ and $V$, we search to reconstruct a binary matrix consistent with these projections or to report that such a matrix does not exist. It is well known that this problem is polynomial [18] (Figure 1). However, it becomes NP-complete by imposing a maximal length of sequence of zeros between the consecutive ones [19], i.e., we impose a maximal number of zeros between two consecutive ones.

\section{WSN Scheduling with Given Number of Visits per Period and Site SSHV(a,b)}

We consider the following problem $\operatorname{SSHV}(\mathrm{a}, \mathrm{b})$ of sensors scheduling. There are $\mathrm{m}$ sites to observe $s_{i}, i \in$ $\{1, \ldots, m\}$, a set of $m$ sensors and a scheduling horizon (interval) $T$ composed of n periods $T=\{1, \ldots, n\}$. During period $t_{j}$ of the time interval $T$, exactly $v_{j}$ sites should be observed by $v_{j}$ sensors. Each site $s_{i}$, should be observed during $h_{i}$ periods. When site $s_{i}$ is not observed at period $t_{j}$, a non visiting penalty $a_{i j}$ is incurred and an information loss penalty $b_{i j} l_{i j}$ is also incurred. We suppose that $a_{i j}$ and $b_{i j}$ are given non negative and $l_{i j}$ is the number of elapsed periods since last visiting site $s_{i}$. Note that the information loss penalty is proportional to the time interval when the site is not observed. The problem now is to determine a surveillance schedule, i.e., to decide for each period which site to observe minimizing the maximum non visiting penalties and information loss penalties.

The problem $\operatorname{SSHV}(\mathrm{a}, \mathrm{b})$ can be reformulated as a mixed integer linear program inspired from [11]. We introduce the binary decision variables $x_{i j}$ and the real variables $y_{i j}$ such that $x_{i j}=1$ and if the site $s_{i}$ is observed at time slot $\mathrm{j}$. The real variable $y_{i j}$ represents the last time

$$
P=\left\{\begin{array}{l}
\min C \\
\text { s.t. } \\
C \geq a_{i j}\left(1-x_{i j}\right)+b_{i j}\left(\mathrm{j}-y_{i j}\right), \quad \forall i ; \forall j \\
\sum_{i=1}^{m} x_{i j}=v_{j}, \quad \forall j \\
\sum_{j=1}^{n} x_{i j}=h_{i}, \quad \forall i \\
0 \leq y_{i j}-y_{i, j-1} \leq j x_{i j}, \quad \forall i ; \forall j \\
j x_{i j} \leq y_{i j} \leq j, \quad \forall i ; \forall j \\
y_{i 1}=0, \quad \forall i \quad \\
x_{i j} \in\{0,1\}, y_{i j} \in R
\end{array}\right.
$$

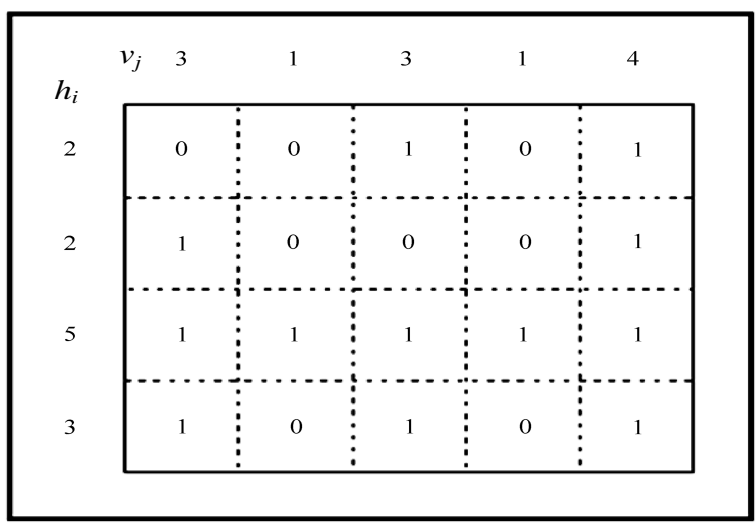

Figure 1. A binary matrix with horizontal projection $H=(2$, $2,5,3)$ and vertical projection $V=(3,1,3,1,4)$; maximal length of sequences of zeros is 3 .

where site $s_{i}$ was observed before period $j$. This gives us the following MIP model:

The constraint (1) ensures that the objective function (C) consists in minimizing the maximum penalty. The constraint (2) ensures that in each period $t_{j}$ there are $v_{j}$ visited sites. The constraints (3) ensure that each site $s_{i}$ is visited in $h_{i}$ periods. Constraints (4) and (5) together ensure that $y_{i j}$ is modified only if site $s_{i}$ is visited $\left(x_{i j}=1\right)$ and takes the value $j$. Constraint (6) implies that all the sites are not observed before the first period.

A solution to the problem $\operatorname{SSHV}(a, b)$ will be presented by an $m \times n$ binary matrix A such that $a_{i j}=1$ only if site $s_{i}$ is visited at period $t_{j}$.

Consider the subproblem $\operatorname{SSHV}(0,1)$ with $a_{i j}=0$ and $b_{i j}=1$ for all the sites over all the periods. Thus for each couple of site and period $\left(s_{i}, t_{j}\right)$ the total penalty is $\left(j-y_{i j}\right)$ which is the number of consecutive non visiting periods before time slot $t_{j}$. If we consider the schedule as a binary matrix, the total penalty is exactly the length of the sequence of zeros before the entry $(i, j)$. Hence the subproblem $\operatorname{SSHV}(0,1)$ is equivalent to reconstructing a binary matrix with horizontal projection $\mathrm{H}$ and vertical projection $\mathrm{V}$ and minimizing the length of the longest sequence of zeros which is NP-complete ( see Section 2). So the general problem $\operatorname{SSHV}(a, b)$ is also NP-complete.

\section{Proposition 1}

The problem $\operatorname{SSHV}(a, b)$ is NP-complete.

\section{WSN Scheduling with a Given Number of Visits per Period}

In this scheduling problem, we search for a sensor scheduling respecting the periodically numbers of visits, i.e., in period $\mathrm{j}$ there are $v_{j}$ visits while minimizing the maximal penalty. The problem $\operatorname{SSV}(a, b)$ can be considered as a relaxation of the problem $\operatorname{SSHV}(a, b)$ where the number of visits for each site is omitted. Yavuz and Jeffcoat [11] studied an NP-complete problem very close to $\operatorname{SSV}(a, b)$. 
They supposed that in each period, at most $\mathrm{m}$ sites was observed instead of exactly $v_{j}$ sites as in $\operatorname{SSV}(a, b)$. In this section, we show that the problem $\operatorname{SSV}(0,1)$ can be solved by a polynomial time algorithm.

As in the previous section, a solution to the problem $\operatorname{SSV}(0,1)$ will be presented by an $m \times n$ binary matrix A such that $a_{i j}=1$ only if site $s_{i}$ is visited at period $t_{j}$. Thus solving $\operatorname{SSV}(0,1)$ with vector of visits $\mathrm{V}$ is equivalent to reconstructing a binary matrix $m \times n$ respecting the vertical projection $\mathrm{V}$ and minimizing the maximal distance. We recall that the distance is the length of a sequence of consecutive zeros. We propose the following cyclic algorithm to solve $\operatorname{SSV}(0,1)$ where the rows are associated to the sites and the columns are associated to the periods.

\begin{tabular}{l}
\hline Algorithm A-SSV \\
\hline - Assign a number from 1 to $\mathrm{m}$ to each site \\
- rows \\
Assign the visits on column 1 to the first $\mathrm{v}_{1}$ \\
rows \\
This process is continued cyclically with row \\
1 being treated as the next row after row $\mathrm{m}$. \\
\hline
\end{tabular}

We state the following result:

\section{Proposition 2}

The algorithm A-SSV solves SSV $(0,1)$ in $\mathrm{O}(m n)$.

Proof The algorithm A-SSV provides a solution S* respecting the vertical projection since at each step $j v_{j}$ ones are assigned to the rows.

Let us show that $\mathrm{S}^{*}$ minimizes the maximal distance. Suppose that in $\mathrm{S}^{*}$, the maximal distance is reached between two 1s placed on cells $(i, j)$ and $(i, j ')$ with $j<j$ '. Since on row $i$, there is no 1 placed between columns $j+1$ and $j^{\prime}-1$ then $\sum_{k=j}^{j^{\prime}-1} v_{k}<m$. If $\mathrm{S}^{*}$ is not optimal then there exists a solution $\mathrm{S}$ with a maximal distance less than that of $\mathrm{S}^{*}$. On each row of $\mathrm{S}$, there is at least an 1 placed between columns $j+1$ and $j^{\prime}-1$. Hence $\sum_{k=j}^{j^{\prime}-1} v_{k} \geq m$, contrary with $\sum_{k=j}^{j^{\prime}-1} v_{k}<m$.

Note that in both addressed problems $\operatorname{SSV}(0,1)$ and $\operatorname{SSHV}(a, b)$, once the schedule is established, the visits should be cyclically assigned to the sensors to improve the lifetime of WSN.

\section{Conclusions}

In this paper, we have studied the wireless sensor network scheduling problem. We have proved that scheduling with a given number of visits for each site and each period with a minimum maximal penalty is an NP-complete problem. We have also proposed a polynomial time algorithm to schedule sensors under only a given number of visits for each period. The problems studied in this paper belong to a rich and relatively unexplored area. Investigating the problems under more real constraints and designing heuristic to solve the hard problems are possible research directions.

\section{References}

[1] A. Sorokin, N. Boyko, V. Boginski, S. Uryasev and P. M. Pardalos, "Mathematical Programming Techniques for Sensor Networks,” Algorithms, Vol. 2, 2009, pp. 565-581.

[2] K. Wu, C. Liu, J. Pan and D. Huang, "Robust Range-Free Localization in Wireless Sensor Networks,” Mobile Networks \& Applications, Vol. 12, No. 5, 2007, pp. 392-405.

[3] C. Wang and L. Xiao, "Sensor Localization in Concave Environments," ACM Transactions on Sensor Networks, Vol. 4, No. 1, 2008, pp. 1-31.

[4] D. Koutsonikolas, S. M. Das and Y. C. Hu, "Path Planning of Mobile Landmarks for Localization in Wireless Sensor Networks,” Computer Communications, Vol. 30, No. 13, 2007, pp. 2577-2592.

[5] M. Rudafshani and S. Datta, "Localization in Wireless Sensor Networks," Proceedings of the 6th International Conference on Information Processing in Sensor Networks, New York, 25 April 2007, pp. 51-60.

[6] J. Jeong, S. Sharafkandi and D. H. C. Du, "Energy-Aware Scheduling with Quality of Surveillance Guarantee in Wireless Sensor Networks," Proceedings of the 2006 Workshop on Dependability Issues in Wireless Ad Hoc Networks and Sensor Networks, New York, 25 September 2006, pp. 55-64.

[7] K. Wu, Y. Gao, F. Li and Y. Xiao, "Lightweight Deployment-Aware Scheduling for Wireless Sensor Networks,” Mobile Networks \& Applications, Vol. 10, No. 6, 2005, pp. 837-852.

[8] S. S. Singh, N. Kantas, B. N. Vo, A. Doucet and R. J. Evans, "Simulation-Based Optimal Sensor Scheduling with Application to Observer Trajectory Planning," $\mathrm{Au}$ tomatica, Vol. 43, No. 5, 2007, pp. 817-830.

[9] Klappenecker, H. Lee and J. L. Welch, "Scheduling Sensors by Tilinglattices," Proceedings of the 27th ACM Symposium on Principles of Distributed Computing, New York, 11 June 2008, pp. 437-437.

[10] M. Yavuz and D. Jeffcoat, "Single Sensor Scheduling for Multi-Site Surveillance,” Technical Report, Air Force Research Laboratory, Maui, 2007.

[11] M. Yavuz and D. Jeffcoat, "An Analysis and Solution of the Sensor Scheduling Problem," Proceedings of the 7th International Conference on Cooperative Control and Optimization, Gainesville, 31 January-2 February 2007.

[12] C. Commander, P. Pardalos, V. Ryabchenko and S. Uryasev, "The Wireless Network Jamming Problem," Journal of Combinatorial Optimization, Vol. 14, No. 1, 2007, pp. 481-498.

[13] C. Commander, P. Pardalos, V. Ryabchenko, S. Sarykalin, 
T. Turko and S. Uryasev, "Robust Wireless Network Jamming Problems," Lecture Notes in Control and Information Sciences, Springer, Berlin, 2008.

[14] K. Kalpakis, K. Dasgupta and P. Namjoshi, "Efficient Algorithms for Maximum Lifetime Data Gathering and Aggregation in Wireless Sensor Networks," Computer Networks, Vol. 42, No. 6, 2003, pp. 697-716.

[15] S. Stanczak, M. Wiczanowski and H. Boche, "Theory and Algorithms for Resource Allocation in Wireless Networks," Lecture Notes in Computer Science, Springer, Berlin, 2006.

[16] G. Herman and A. Kuba, “Discrete Tomography: Foun- dations, Algorithms and Applications,” Applied and Numerical Harmonic Analysis, Birkhäuser, Boston, 1999.

[17] G. Herman and A. Kuba, "Advances in Discrete Tomography and its Applications," Applied and Numerical Harmonic Analysis, Birkhäuser, Boston, 2007.

[18] H. J. Ryser, "Combinatorial Properties of Matrices of Zeros and Ones," Canadian Journal of Mathematics, Vol. 9, 1957, pp. 371-377.

[19] F. Jarray, M.-C. Costa and C. Picouleau, "Complexity Results for the Horizontal Bar Packing Problem,” Information Processing Letters, Vol. 108, No. 6, 2008, pp. 356-359. 